\title{
A UTILIZAÇÃO DAS FERRAMENTAS DE GESTÃO APLICADAS ÀS UNIDADES DE INFORMAÇÃO (UI): PERCEPÇÕES DOS GESTORES
}

\begin{abstract}
Resumo: Traz um panorama acerca da utilização de ferramentas de gestão por gestores de Unidades de Informação em seus contextos profissionais, a partir de uma pesquisa exploratória executada em três fases: a primeira identifica o potencial de utilização das ferramentas de gestão de Unidades de Informação, baseando-se na produção científica e técnica; a segunda constrói um questionário para ser aplicado entre gestores de Unidades de Informação; e a terceira analisa os dados obtidos nos questionários aplicados. Os resultados estão estruturados na seguinte ordem: 1) Área de formação dos gestores; 2) Ferramentas de gestão conhecidas pelos gestores das Unidades de Informação; 3) Ferramentas de gestão utilizadas pelas Unidades de Informação; e 4) Resultados positivos alcançados em decorrência do uso das ferramentas. Quando questionados sobre as ferramentas que conheciam, as respostas dos gestores mostraram que a Matriz SWOT é a mais conhecida, com 94,4\%; seguida pelo Mapa Conceitual, com 88,9\%; Diagrama de Pareto, com 61,1\%; PDCA, $55,6 \%$, 5w2h, 44,4\% e Business Process Management (BPM), com resultado igual ao $5 \mathrm{w} 2 \mathrm{~h}$. Sobre as mais utilizadas, verificouse o quadro a seguir: Mapa Conceitual $(38,9 \%)$, Matriz SWOT $(33,3 \%), 5 w 2 h(16,7 \%)$ e PDCA $(16,7 \%)$. Chama a atenção que $38,9 \%$ dos gestores nunca utilizaram nenhuma ferramenta. Com isto, nota-se na pesquisa o destaque do Mapa Conceitual e da Matriz SWOT; e a informação que todos os gestores que utilizaram ferramentas de gestão relataram ter obtido algum resultado positivo.
\end{abstract}

Palavras-chave: ferramentas de gestão. Unidades de informação. Gestão e inovação.

\author{
Natanael Vitor Sobral \\ Doutor em Ciência da \\ Informação. UFBA. \\ natanvsobral@gmail.com
}

Iuri Santana Amadeu

Graduando em Arquivologia.

UFBA.

iuri.03santana.amadeu@hotmail

.com

Lindaura Teixeira Santos

Graduanda em Arquivologia.

UFBA.

lindaurateixeiras@gmail.com

Louise Anunciação Fonseca de Oliveira do Amaral

Doutoranda em Ciência da Informação. UFBA.

louise.anunciacao@ufba.br

Tatyane Lúcia Cruz

Mestra em Ciência da Informação. UFPE. tatyanelcruz@gmail.com

\section{THE USE OF MANAGEMENT TOOLS APPLIED TO INFORMATION UNITS (IUs): MANAGER'S PERCEPTIONS}

\begin{abstract}
It provides an overview of the use of management tools by Information Unit managers in their professional contexts, based on an exploratory research carried out in three phases: the first one identifies the potential of using UI management tools based on scientific production. and technique; the second builds a questionnaire to be applied among IU managers; and the third analyzes the data obtained from the applied questionnaires. The results are structured in the following order: 1) Training area of managers; 2) Management tools known to UI managers; 3) Management tools used by the UI; and 4) Positive results achieved as a result of using the tools. When asked about the tools they knew, the managers' answers showed that the SWOT Matrix is the best known, with 94.4\%; followed by the Concept Map, with 88.9\%; Pareto diagram, with 61.1\%; PDCA, $55.6 \%, 5 w 2 h, 44.4 \%$ and Business Process Management (BPM), with a result equal to 5w2h. Regarding the most used, the following table was verified: Concept Map (38.9\%), SWOT Matrix (33.3\%), 5w2h (16.7\%) and PDCA $(16.7 \%)$. It is noteworthy that $38.9 \%$ of managers never used any tool. Thus, the research highlights the concept map and the SWOT matrix; and the information that all managers using management tools reported had a positive result.
\end{abstract}

Keywords: management tools. Information units. Management and innovation. 


\section{INTRODUÇÃO}

O tema inovação é recorrente na literatura científica, em especial, quando se trata do contexto organizacional. Para as empresas, a inovação é um elemento imprescindível que contribui para o aumento da competitividade, ajudando-as a sobreviver em mercados cada vez mais disputados. No âmbito das Unidades de Informação (UI) brasileiras, este tema ainda é subestimado. Em trabalho recente, Sobral, Calheira e Ferreira (2017) perceberam que há um conjunto significativo de publicações indexadas na base de informação Web of Science sobre o assunto, sendo estas as localidades mais representativas: Pequim, China (6 publicações), Vitória, Austrália (6), Nova Brunswick, Estados Unidos (4), Pretória, África do Sul (4), Queensland, Austrália (4), Teerã, Irã (4), Tucson, Estados Unidos (4). Ressalta-se que outros países do continente europeu também figuraram positivamente neste levantamento, tais como Inglaterra e Espanha. Deste modo, verifica-se que o Brasil ainda tem um longo caminho a percorrer no que concerne à concepção e implementação de ações inovadoras no contexto das UI, sobretudo, no que pesa à produção científica internacional indexada em bases de dados.

Acredita-se que um dos fatores que indicam o atraso do Brasil neste tema é a falta de investimentos e a ausência de uma ambiência inovadora mais aguçada no país. Quanto às UI brasileiras, além da falta de senso de competitividade, estas estão fortemente vinculadas e dependentes do contexto público, que padece cada vez mais de falta de recursos e incentivos à inovação no âmbito da gestão pública. Quanto à ambiência inovadora, percebe-se que este não é apenas um problema das UI, de um modo geral, o país está mal colocado no ranking mundial de inovação. Segundo a Organização Mundial de Propriedade Intelectual (OMPI), o país ocupa o $64^{\circ}$ lugar no ranking que afere este quesito.

Diante deste contexto e visando fomentar a inovação em UI, elaborou-se um projeto intitulado "Tendências de gestão e inovação em UI", em desenvolvimento no âmbito do Grupo de Estudos de Políticas de Informação, Comunicações e Conhecimento (Gepicc), do Instituto de Ciência da Informação da Universidade Federal da Bahia (ICI/UFBA). Esta iniciativa iniciou-se nas disciplinas ICI002 e ICIA39, intituladas "Administração de UI", do Departamento de Fundamentos e Processos Informacionais do ICI/UFBA, que são ministradas para os cursos de graduação em Biblioteconomia e Documentação e Arquivologia. Com isto, espera-se desenvolver mindsets inovadores nos alunos, para que estes sejam capazes de 
transformar positivamente as bibliotecas, arquivos, museus, memoriais, editoras, livrarias, sebos, centros de documentação e de informação, em suas atuações profissionais.

As dimensões presentes no projeto são as seguintes:

a) Produção Científica: visa refletir sobre conceitos e teorias relacionadas às UI. Envolve levantamento da literatura nacional e internacional sobre o tema e compreensão dos principais autores e referenciais teóricos. Está presente em todo o projeto, de modo transversal, com o propósito de atualização dos pesquisadores envolvidos; b) Produção Técnica: pretende verificar as principais ferramentas existentes na academia e no mercado, úteis para os processos de gestão e inovação no âmbito das UI. Atualmente, essa é a fase em que o projeto se encontra. Com o intuito de compreender a popularidade e utilização destas ferramentas pelos gestores de UI, realizaram-se levantamentos envolvendo a aplicação de questionários; c) Análise in loco: conjunto de atividades que serão realizadas a posteriori, objetivando reconhecer o ambiente das UI, capacitando gestores e profissionais da informação atuantes nestas instituições para lidarem com a temática de gestão e inovação.

Tendo em vista que grande parte das instituições documentais ainda se encontram pautadas em modelos administrativos clássicos, pressupõe-se que no cenário atual há baixa adesão dos gestores à utilização de ferramentas de gestão, que são catalizadoras dos mais diversos tipos de inovação, a saber: inovação de marketing, de produto/serviço, de processo e organizacional, categorização adotada no manual de Oslo da Organização para a Cooperação e Desenvolvimento Econômico (OCDE). Assim, tem-se como pressuposto que a utilização de ferramentas pode impulsionar a inovação nas organizações e isto motivou a realização deste trabalho. Outro importante pressuposto é que as instituições organizadas e profissionalizadas, no aspecto da gestão, possuem mais facilidade de inovar, do que outras que praticam a gestão amadora, pautada em fundamentos mais intuitivos.

Baseando-se nisto, pretende-se aqui apresentar um panorama sobre a utilização de ferramentas de gestão por gestores de UI em seus contextos profissionais, descrevendo-o e conhecendo os resultados que as ferramentas proporcionam.

\section{REFERENCIAL TEÓRICO}

Segundo o Manual de Oslo da OCDE (2005), inovação é a implementação de um produto (bem ou serviço) novo ou significativamente melhorado, ou um processo, ou um novo 
método de marketing, ou um novo método organizacional nas práticas de negócios, na organização do local de trabalho ou nas relações externas. Para Conto, Antunes Júnior e Vaccaro (2016), a inovação está relacionada à sobrevivência das organizações, sendo a sua principal estratégia competitiva. Jacobi (2006), de modo objetivo, lembra que a inovação se baseia na introdução de mudanças dentro de uma ordem planejada, tendo assim, uma dimensão aplicada.

Para as UI, sendo estas, em sua maioria, organizações do serviço público, a inovação é resultado de políticas públicas bem elaboradas, tendo como resultado a produção de serviços públicos mais eficientes (FRANÇA, 2017). Em âmbito interno, os profissionais da informação que administram as UI podem elaborar uma série de estratégias com o propósito de fomentar a inovação, como por exemplo: introduzir novas práticas profissionais; aprimorar técnicas e processos; melhorar a estrutura física e organizacional; canalizar esforços para pesquisa e desenvolvimento de tecnologias da informação; elaborar estratégias de marketing voltadas para produtos, serviços e relacionamento com seu público, influenciando no reposicionamento da imagem da instituição; entre outras (BRAGANÇA et al., 2016).

Cândido, Bertotti e Bedin (2017), defendem que, além de ser um fator decisivo nos resultados e performance das organizações, a inovação pressupõe a introdução de novas ferramentas e/ou soluções para as mais diversas situações que venham a ser detectadas no ambiente organizacional, em convergência ao que se defende neste trabalho.

A inovação permite que um grande campo de possibilidades se construa, podendo assim, alcançar diferentes dimensões institucionais. A aplicação de práticas inovadoras estimula a ruptura de modelos, que, por muito tempo, consolidaram-se em UI, não sendo objeto de crítica e reformulação, tornando-se obsoletos frente às necessidades e preferências dos usuários atuais. Com isto, os resultados destas instituições foram comprometidos, haja vista que algumas UI deixaram de ter sua relevância percebida pela comunidade devido às transformações tecnológicas, culturais e comportamentais do seu público-alvo.

A gestão, em sua essência, implica em diagnosticar, planejar e controlar um sistema para que alcance resultados satisfatórios. Desta feita, é importante que as funções administrativas sejam orientadas à inovação. Assim, entende-se que a inovação tem potencial de atingir de modo transversal todas as atividades de uma organização, adequando a maneira como a instituição observa o mercado, se organiza para atendê-lo e avalia suas ações junto a ele. No intuito de subsidiar estas ações, as ferramentas de gestão são instrumentos bastante 
conhecidos e utilizados pela academia e o mercado para a concretização das funções administrativas, permitindo a elaboração de estratégias que permitam à organização atingir seus resultados. $\mathrm{O}$ alinhamento entre a gestão e a inovação é fundamental, tendo em vista que ambas possuem funções complementares nos processos organizacionais, sendo, inclusive, as ferramentas de gestão, instrumentos catalisadores da inovação, que favorecem a solução de problemas organizacionais específicos, que envolvem a observação dos ambientes interno e externo, o mapeamento de processos, a gestão de elementos tangíveis e intangíveis, a abertura de novos mercados, o relacionamento com usuários e clientes estabelecidos etc.

\section{METODOLOGIA}

Esta pesquisa contempla as abordagens qualitativa e quantitativa, sendo exploratória quanto aos seus fins, e quanto aos meios, utiliza-se da pesquisa bibliográfica e da aplicação de questionários. O percurso metodológico foi constituído através de três passos.

A primeira etapa foi identificação do potencial da utilização das ferramentas de gestão nas UI, baseando-se na produção científica e técnica da área. Foi nesta fase que foram separadas as ferramentas mais representativas mencionadas nos trabalhos pesquisados para subsidiar a fase seguinte do trabalho.

A segunda fase se deu pela elaboração de um questionário direcionado aos gestores de UI (arquivos, bibliotecas, museus, editoras, memoriais, centros de documentação e de informação). Este questionário buscou identificar como ocorre o uso destas ferramentas de gestão na rotina dos entrevistados no âmbito de suas atividades nas UI. Para tal, foi criado um formulário, com perguntas abertas e de múltipla escolha, visando responder questões relacionadas a: formação dos entrevistados, conjunto de ferramentas conhecidas, utilizadas e seus respectivos benefícios. Estas perguntas foram enviadas em forma de questionário eletrônico para o e-mail dos gestores ao longo do primeiro semestre de 2019 . No total, obtevese a contribuição de 18 respondentes. Foram considerados gestores, todos aqueles que possuíam algum cargo ligado à supervisão, coordenação, direção, chefia, formalmente ou informalmente, exercendo funções de responsabilidade no ambiente das UI.

O terceiro momento da pesquisa foi o de analisar os dados obtidos. Visando aprofundar os resultados para trabalhos futuros, o questionário ainda se encontra disponível para o recebimento de respostas objetivando a realização de uma pesquisa futura mais ampla. Os 
contatos dos respondentes foram levantados em grupos profissionais, de conselhos, associações e em listas de e-mails oriundas de congressos científicos, sem limitação geográfica dentro do território nacional (Brasil).

\section{RESULTADOS E DISCUSSÃO}

Os resultados estão estruturados na seguinte ordem: 1) Área de formação dos gestores; 2) Ferramentas de gestão conhecidas pelos gestores das UI; 3) Ferramentas de gestão utilizadas pela UI; e 4) Resultados positivos alcançados em decorrência do uso das ferramentas.

Como ponto de partida, os gestores responderam quais eram as suas áreas de formação no nível graduação. Nos resultados, nota-se que alguns respondentes possuem mais de uma graduação, e por isso, marcaram mais de uma opção, totalizando 21 itens. Com isto, no Gráfico 1 , verifica-se que ocorreu um empate, $50,0 \%$ (9) dos gestores consultados possuem formação em Biblioteconomia, enquanto outra metade é formada em Arquivologia. As demais opções foram menos sinalizadas pelos gestores, evidenciando que 5,6\% (1) dos entrevistados também são formados em cursos como Comunicação, Direito e Relações Públicas e Marketing.

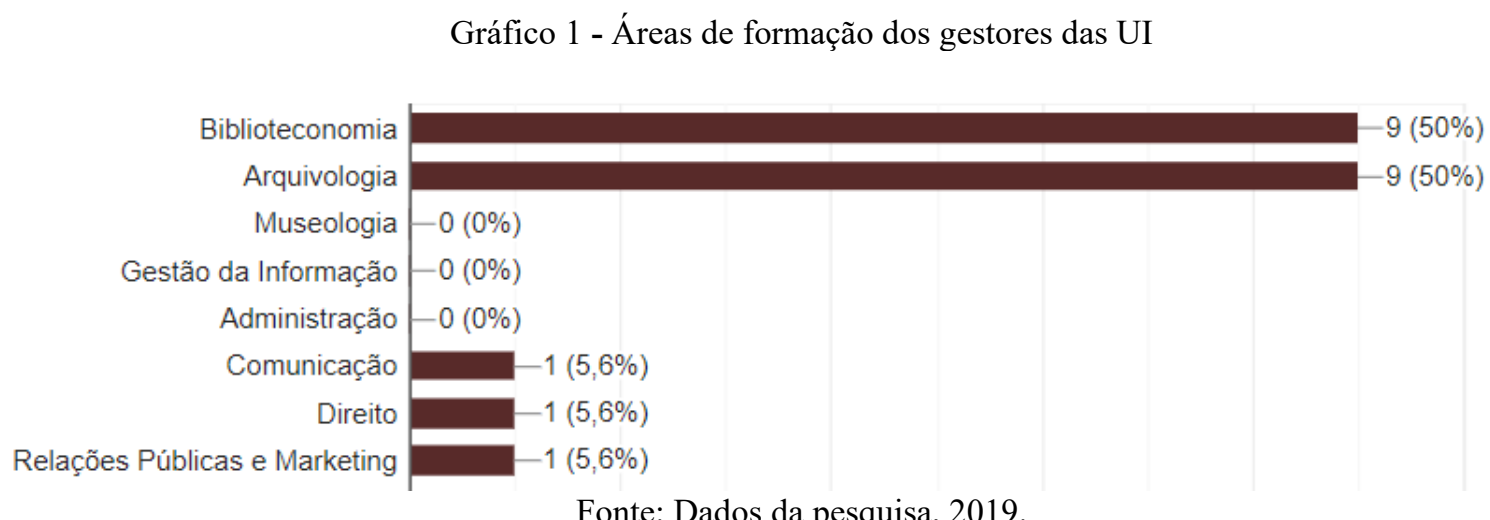

Fonte: Dados da pesquisa, 2019.

Ao questionar em quais instituições estes gestores trabalham verificou-se que apenas 1 atua em âmbito privado e 17 em organizações públicas, o que confirma a natureza pública e social da atividade. Deste modo, nota-se que, é muito difícil dissociar as discussões sobre UI do debate sobre gestão e inovação no setor público, tendo em vista que parte significativa deste pessoal integra o primeiro setor. 
Quando questionados sobre as ferramentas que conheciam, as respostas dos gestores mostraram que a Matriz SWOT é a mais conhecida, com 94,4\% (17); seguida pelo Mapa Conceitual, com 88,9\% (16); Diagrama de Pareto, com 61,1\% (11); PDCA, 55,6\% (10), 5w2h, 44,4\% (8) e Business Process Management (BPM), com resultado igual ao 5w2h (Gráfico 2).

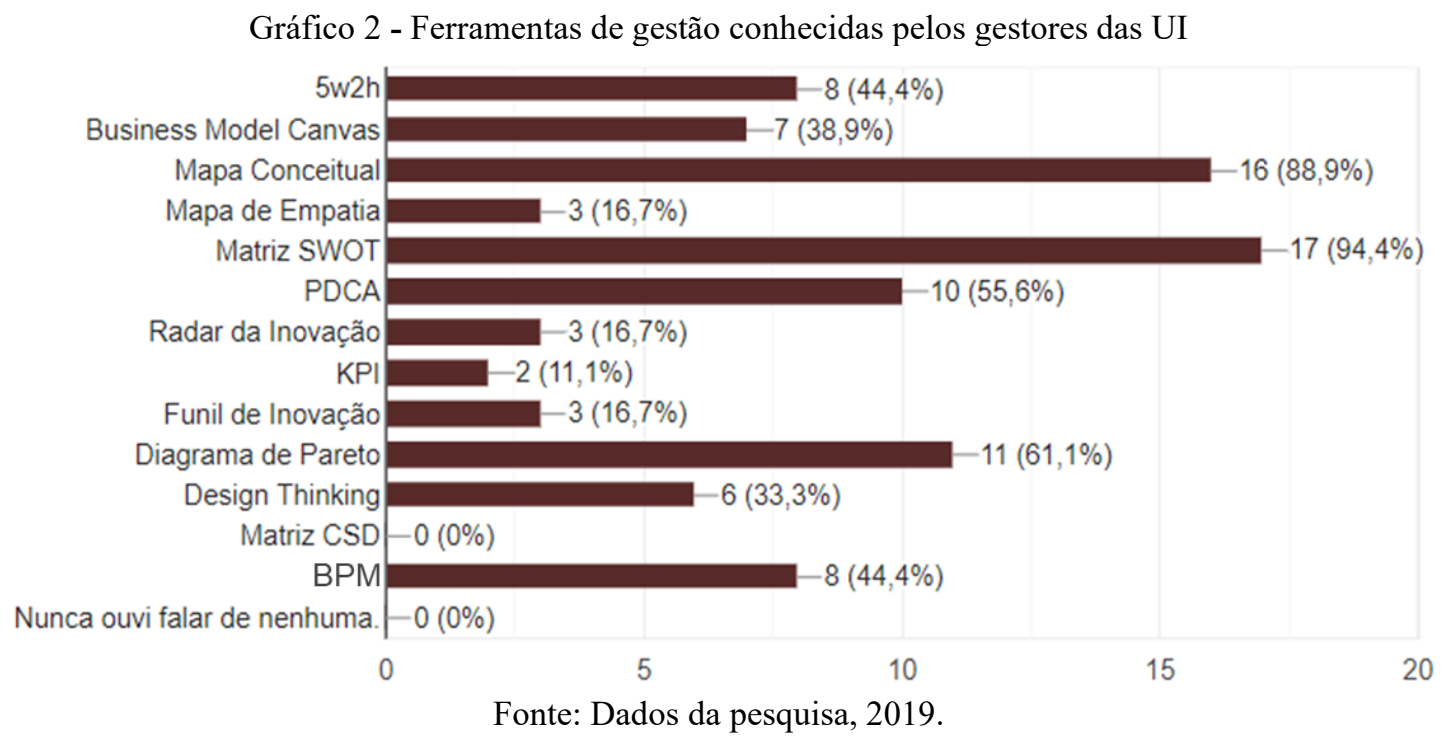

As ferramentas mais recorrentes têm sua utilização centrada nas funções administrativas de diagnóstico e planejamento, mas possuem dinâmicas diferentes. A Matriz SWOT possibilita a realização de diagnósticos na instituição, destacando as suas características, de acordo com os itens: pontos fortes - strengths (S), pontos fracos - weaknesses (W), oportunidades opportunities $(\mathrm{O})$ e ameaças - threats $(\mathrm{T})$. Os pontos fortes e fracos dizem respeito ao ambiente interno, sendo consideradas variáveis controláveis, enquanto as oportunidades e ameaças podem ser traçadas observando o ambiente externo, e por isso, são categorizadas como variáveis incontroláveis. Quanto ao Mapa Conceitual, devido à sua versatilidade, pode ser construído de maneira unidimensional ou bidimensional (SOUZA; BORUCHOVITCH, 2010). O Mapa Conceitual é mais utilizado nas fases de diagnóstico e planejamento, tomando por base os fluxos de pensamento dos seus realizadores, que criam um mapa de relações entre conceitos que pode ser útil para a identificação de um problema, sua solução ou para a caracterização de algo que se pretende entender ou explicar. Sob o aspecto da aplicação, estas ferramentas podem ser utilizadas de maneira analógica, fazendo uso de papel, murais e caneta, ou então, amparando-se em algum recurso tecnológico, como softwares e sites que ajudam a sintetizar as categorias e fornecem explicação sobre o emprego da ferramenta. 
Em seguida, buscou-se expressar as ferramentas de gestão conhecidas pelos gestores, mas que não estavam listadas nas alternativas do questionário. Com isto, constatou-se, que: Kanban, Business Intelligence (BI), Balanced Scorecard e o Brainstorming foram as mais indicadas. Deste modo, obteve-se um conjunto adicional de ferramentas para compor as categorias pré-definidas em pesquisas futuras.

Usado como ferramenta de controle, o Kanban é um sistema de controle da produção desenvolvido no Japão, em que sua principal função é regular os níveis de estoque (SERENO et al., 2011). Baseia-se em cartões e sinalizações que indicam as fases da produção, favorecendo o monitoramento das atividades. O BI é utilizado para a ampliação da inteligência de negócios das organizações, determinando a maneira como a informação é coletada, processada e utilizada, tornando conjunto de dados aparentemente desconexos em informações úteis para tomada de decisão em organizações. O Balanced Scorecard apresenta um modelo de gestão estratégica com o foco do planejamento financeiro, por meio da ferramenta é possível construir indicadores organizacionais ligados às múltiplas atividades e funções institucionais distribuídas nas áreas: financeira, processos internos, clientes, aprendizado e crescimento. O Brainstorming, em suma, é um método em que são anotadas na fase inicial de um planejamento, todas as ideias possíveis, e depois é feita uma seleção das melhores que podem ser aplicadas no ambiente.

Os gestores indicaram conhecer 12 ferramentas dentre as categorias pré-definidas, e além disto, contribuíram acusando ter ciência de mais 4. A única ferramenta apresentada e apontada como não conhecida foi a matriz CSD (Certezas, Suposições e Dúvidas), que ajuda a iniciar processos de diagnóstico e planejamento de um determinado projeto, nivelando o conhecimento entre os atores envolvidos.

Adiante, no Gráfico 3, estão os resultados relativos às ferramentas já utilizadas nos pelos gestores respondentes no âmbito das UI em que exercem função gerencial. 


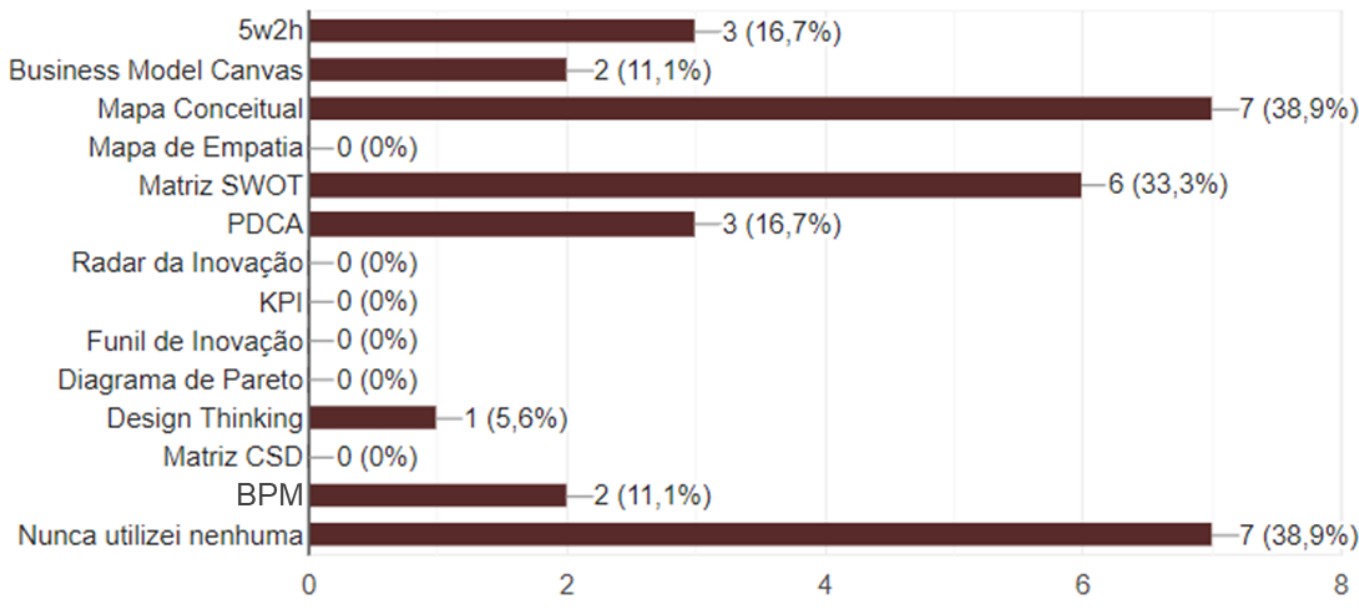

Fonte: Dados da pesquisa, 2019.

Ao comparar o Gráfico 3 com o Gráfico 2, observa-se que a ferramenta mais conhecida (Matriz SWOT), não é a mais utilizada, sendo o Mapa Conceitual mais proeminente neste quesito, ainda que por uma diferença bem pequena. Dessa forma, a segunda mais conhecida (Mapa Conceitual), toma o primeiro lugar no quesito utilização. Apesar de o Mapa Conceitual e da Matriz SWOT apresentarem uma boa quantidade de utilizadores, o número de respostas alcançado pelos que declaram nunca ter utilizado nenhuma das ferramentas $(38,9 \%)$ empata com o Mapa Conceitual e supera a Matriz SWOT (33,3\%), indicando que há um quantitativo importante de gestores que nunca utilizou nenhuma das ferramentas apresentadas. Esse dado, permite inferir, que há uma baixa correlação entre "conhecimento" e "utilização", haja vista que nenhum respondente declarou "nunca ter ouvido falar" das ferramentas apresentadas (Gráfico 2), porém, 38,9\% nunca utilizou nenhuma ferramenta (Gráfico 3).

Dentre as ferramentas que tiveram a utilização zerada no questionário, sendo assim, nunca foram utilizadas pelos gestores que responderam o inquérito, nota-se que estas são relacionadas aos seguinte tópicos: (a) Estudo de Usuário, representado no mal resultado do mapa de empatia; (b) Indicadores, expressado no resultado fraco de KPI e Diagrama de Pareto; (c) Inovação, indicado pelo resultado negativo do Radar da Inovação e Funil de Inovação; e (d) Gestão do Conhecimento, presente no desconhecimento sobre a matriz CSD, que conforme ilustrado no gráfico 2, não é conhecida, e por isso, nunca foi utilizada. Sobre as ferramentas não citadas nos itens pré-definidos, porém utilizadas pelos gestores, destacaram-se: Kanban, BI e Brainstorming, livremente citadas pelos indivíduos quando questionados se existe alguma 
ferramenta de gestão não mencionada na pergunta anterior que você utilizou e deseja mencionar?

No tópico seguinte, quando perguntados sobre a ocorrência de resultados positivos advindos do uso de alguma dessas ferramentas, 61,1\% (11) dos gestores informaram ter obtido bons resultados ao utilizá-las, sendo isto demonstrado no Gráfico 4. Considerando que 38,9\% declararam nunca ter utilizado nenhuma ferramenta (Gráfico 3), o que indica que 61,1\% utilizaram, confrontam-se estes resultados com o Gráfico 4 e percebe-se que, todos os gestores $(61,1 \%)$ que utilizaram ferramentas obtiveram algum resultado positivo, o que demonstra o poder das ferramentas como impulsionadoras da gestão e da inovação em ambientes organizacionais.

Gráfico 4 - Resultados positivos alcançados em decorrência do uso das ferramentas

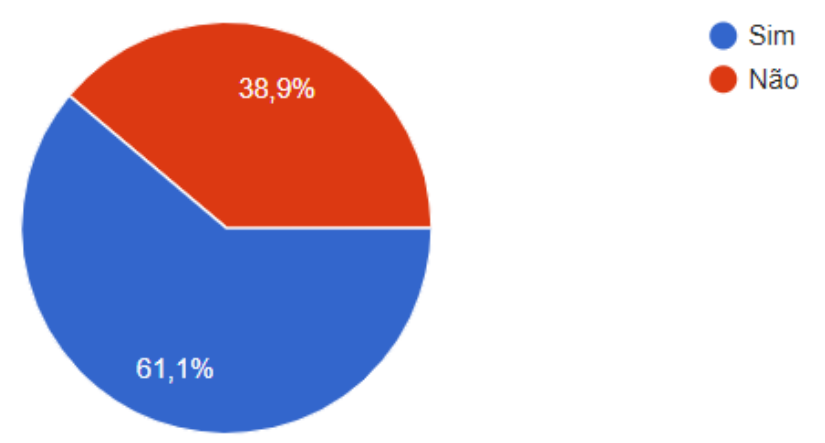

Fonte: Dados da pesquisa, 2019.

Com o propósito de aprofundar estes achados, perguntou-se aos respondentes quais foram os resultados positivos relacionados ao uso dessas ferramentas, em questão aberta, e as respostas mais comuns, em ordem de representatividade, foram as seguintes: $\left(1^{\circ}\right)$ melhoria na gestão de projetos, $\left(2^{\circ}\right)$ marketing, e por fim, $\left(3^{\circ}\right)$ motivação dos colaboradores e recursos humanos, financeiro, autoconhecimento, atendimento, processos, produtividade, qualidade e indicadores, o que demonstra a alta versatilidade das ferramentas e dá indícios sobre seus contextos mais profícuos de utilização. 


\section{CONSIDERAÇÕES FINAIS}

Procurou-se apresentar aqui um panorama acerca da utilização de ferramentas de gestão por gestores de UI em seus contextos profissionais, colaborando dessa forma na descrição dos cenários encontrados com a realização da pesquisa. Logo, foi possível conhecer aspectos peculiares dos resultados que as ferramentas de gestão proporcionam.

Assim sendo, viu-se que numerosas são as ferramentas conhecidas por gestores de UI, contudo, esse conhecimento não se traduz necessariamente no uso dessas em seus ambientes de trabalho. Apesar de conhecerem variadas ferramentas, aproximadamente 39\% dos gestores informaram nunca terem utilizado estes recursos em seus contextos profissionais, o que demonstra a existência de um grande campo ainda necessitado de estímulos inovadores no que se refere a práticas de gestão.

Gestores que conhecem e utilizam as ferramentas de gestão nas UI, apontaram os ganhos em decorrência dessa prática, relacionados, em especial, à gestão de projetos, destacando também a versatilidade destas ferramentas no ambiente da gestão. O Mapa Conceitual e a Matriz SWOT foram as ferramentas com maior destaque quanto ao uso no contexto analisado.

A partir das identificações observadas por meio dos resultados obtidos, visa-se então uma nova fase do projeto, a realização de uma análise in loco nas UI que focará na realização de atividades intentando capacitar a equipe profissional para lidar com a gestão de maneira mais profissionalizada e integrada à inovação.

\section{REFERÊNCIAS}

BRAGANÇA, F. F. C. et al. Marketing, criatividade e inovação em unidades de informação. Revista Brasileira de Marketing, São Paulo, v. 15, n. 2, p. 237-245, 2016.

CÂNDIDO, A. C.; BERTOTTI, P. S. S; BEDIN, J. O potencial das ferramentas atuais de Gestão \& Negócios aplicados às Unidades de Informação. Revista Brasileira de Biblioteconomia e Documentação, v. 13, p. 1165-1182, 2017.

CONTO, S. M.; ANTUNES JÚNIOR, J. A. V.; VACCARO, G. L. R. A inovação como fator de vantagem competitiva: estudo de uma cooperativa produtora de suco e vinho orgânicos. Gestão \& Produção, São Carlos, v. 23, n. 2, p. 397-407, 2016. Disponível em: 
http://www.scielo.br/scielo.php?pid=S0104-

530X2016005002103\&script=sci abstract\&tlng=pt. Acesso em: 08 ago. 2019.

FRANÇA, A. M. B. A inovação no setor público. Cadernos da Escola Paulista de Contas

Públicas, v. 1, n. 1, p. 10-14, 2017. Disponível em: https://www.tce.sp.gov.br/epcp/cadernos/index.php/CM/article/download/3/4. Acesso em: 08 jul. 2019.

JACOBI, P. R.; PINHO, J. A. Inovação no campo da gestão pública local: novos desafios, novos patamares. FGV Editora, 2006.

OCDE. Organização para a Cooperação e Desenvolvimento Econômico. Manual de Oslo: proposta de diretrizes para coleta e interpretação de dados sobre inovação tecnológica. 3. ed. Brasília, DF: FINEP, 2005.

SERENO, B. et al. Método híbrido CONWIP/KANBAN um estudo de caso. Gestão \& Produção, São Carlos, v. 18, n. 3, p. 651-672, 2011. Disponível em: http://www.scielo.br/scielo.php?script=sci_arttext\&pid=S0104530X2011000300015\&lng=en\&nrm=iso. Acesso em: 08 ago. 2019.

SOUZA, N. A.; BORUCHOVITCH, E. Mapas conceituais: estratégia de ensino/aprendizagem e ferramenta avaliativa. Educação em Revista, Belo Horizonte, v. 26, n. 3, p. 195-218, 2010. Disponível em: http://www.scielo.br/pdf/edur/v26n3/v26n3a10. Acesso em: 01 jun. 2019. 